\title{
SMOOTH POINTS IN OPERATOR SPACES AND SOME BISHOP-PHELPS-BOLLOBÁS TYPE THEOREMS IN BANACH SPACES
}

\author{
DEBMALYA SAIN
}

\begin{abstract}
We introduce the notion of approximate norm attainment set of a bounded linear operator between Banach spaces and use it to obtain a complete characterization of smooth points in the space of compact linear operators, provided the domain space is reflexive and Kadets-Klee. We also apply the concept to characterize strong BPB property (sBPBp) of a pair of Banach spaces. We further introduce uniform $\varepsilon-\mathrm{BPB}$ approximation of a bounded linear operator and uniform strong BPB property (uniform sBPBp) with respect to a given family of norm one linear operators and explore some of the relevant properties to illustrate its connection with earlier studies on Bishop-Phelps-Bollobás type theorems in Banach spaces. It is evident that our study has deep connections with the study of smooth points in operator spaces. We obtain a complete characterization of uniform sBPBp for a pair of Banach spaces, with respect to a given family of norm one bounded linear operators between them. As the final result of this paper, we prove that if $\mathbb{X}$ is a reflexive Kadets-Klee Banach space and $\mathbb{Y}$ is any Banach space, then the pair $(\mathbb{X}, \mathbb{Y})$ has sBPBp for compact operators. Our results extend, complement and improve some of the earlier results in this context.
\end{abstract}

Mathematics subject classification (2010): 46B20, 46B04, 47L05. operators.

Keywords and phrases: Banach space, norm attainment, Bishop-Phelps-Bollobás property, smooth

\section{REFERENCES}

[1] Acosta, M. D., Aron, R. M., Garcia, D., Maestre, M., The Bishop-Phelps-Bollobás theorem for operators, Journal of Functional Analysis, 254 (2008), 2780-2799.

[2] Bishop, E., Phelps, R. R., A proof that every Banach space is subreflexive, Bull. Amer. Math. Soc., 67 (1961), 97-98.

[3] Bollobás, B., An extension to the theorem of Bishop and Phelps, Bull. London Math. Soc., 2 (1970), 181-182.

[4] Dantas, S., On the Bishop-Phelps-Bollobás type theorems, Doctoral thesis, Valencia University, 2017.

[5] Dantas, S., Some kind of Bishop-Phelps-Bollobás property, Math. Nachr., 290, Issue 5-6, 2017, 774-784.

[6] Dantas, S., Kadets, V., Kim, S. K., Lee, H. J., Martin, M., There is no operatorwise version of the Bishop-Phelps-Bollobás property, Linear Multilinear Algebra, 2018, doi.org/10.1080/03081087.2018.1560388.

[7] PolaK, T., Sims, B., A Banach space which is fully 2-rotund but not locally uniformly rotund, Canad. Math. Bull., 26(1), 1983, 118-120.

[8] SAIN, D., On the norm attainment set of a bounded linear operator, J. Math. Anal. Appl., 457, 2018, 67-76.

[9] Paul, K., Sain, D., Ghosh, P., Birkhoff-James orthogonality and smoothness of bounded linear operators, Linear Algebra Appl., 506, 2016, 551-563. 